\title{
Feedback, receptor clustering, and receptor restriction to single cells yield large Turing spaces for ligand-receptor-based Turing models
}

\author{
Tamás Kurics, ${ }^{1, *}, \dagger$ Denis Menshykau, ${ }^{1,2, *}$ and Dagmar Iber ${ }^{1,2, \ddagger}$ \\ ${ }^{1}$ Department for Biosystems Science and Engineering, ETH Zurich, Mattenstrasse 26, 4058 Basel, Switzerland \\ ${ }^{2}$ Swiss Institute of Bioinformatics (SIB), Switzerland \\ (Received 15 March 2014; revised manuscript received 7 June 2014; published xxxxxx)
}

\begin{abstract}
Turing mechanisms can yield a large variety of patterns from noisy, homogenous initial conditions and have been proposed as patterning mechanism for many developmental processes. However, the molecular components that give rise to Turing patterns have remained elusive, and the small size of the parameter space that permits Turing patterns to emerge makes it difficult to explain how Turing patterns could evolve. We have recently shown that Turing patterns can be obtained with a single ligand if the ligand-receptor interaction is taken into account. Here we show that the general properties of ligand-receptor systems result in very large Turing spaces. Thus, the restriction of receptors to single cells, negative feedbacks, regulatory interactions among different ligand-receptor systems, and the clustering of receptors on the cell surface all greatly enlarge the Turing space. We further show that the feedbacks that occur in the FGF10-SHH network that controls lung branching morphogenesis are sufficient to result in large Turing spaces. We conclude that the cellular restriction of receptors provides a mechanism to sufficiently increase the size of the Turing space to make the evolution of Turing patterns likely. Additional feedbacks may then have further enlarged the Turing space. Given their robustness and flexibility, we propose that receptor-ligand-based Turing mechanisms present a general mechanism for patterning in biology.
\end{abstract}

DOI: 10.1103/PhysRevE.00.002700

PACS number(s): 87.10.Ca, 05.65.+b, 87.17.Pq, 87.10.Kn

\footnotetext{
*These authors contributed equally. http://www.bsse.ethz.ch/cobi/

${ }^{\dagger}$ Present address: Institute of Cognitive Neuroscience and Psychology, Research Centre for Natural Sciences, Hungarian Academy of Sciences, Budapest, Hungary.

${ }^{\ddagger}$ dagmar.iber@bsse.ethz.ch
}

it has been suggested that differences in diffusion speed may 47 arise from transient differences in the interactions with the 48 extracellular matrix [12]. A number of theoretical studies 49 seek to overcome the requirement of a large difference in 50 diffusivity of Turing components, and an emergence of Turing 51 pattern has been shown to be possible also in the presence of a 52 single diffusive specie coupled to a quenched oscillator [13]; 53 cell migration rather than diffusion has been proposed to 54 result in Turing instabilities [14,15]. Finally, cross-diffusion 55 and nonlinear diffusion have been shown to support the 56 formation of Turing-type patterns, such that Turing patterns 57 can arise for any ratio of the main diffusivities [16-21]. Cross- 58 diffusion has been shown to arise in crowded environments 59 with finite carrying capacity, i.e., if diffusion is limited 60 when local concentrations or densities reach the carrying 61 capacity $[20,22]$.

Another problem with the applicability of Turing mech- 63 anisms to biological pattern formation concerns the size of 64 the parameter space that gives rise to Turing patterns, the 65 Turing space. This parameter space is small for all known 66 Turing mechanisms in the sense that kinetic parameters can 67 be varied only a few fold as long as physiological constraints 68 on the kinetic constants and relative diffusion constants are 69 respected [24]. It is therefore unclear how evolution could have 70 produced such a mechanism in the first place and how it could 71 have been reused in different settings during the evolution 72 of new species. Moreover, biological systems are noisy, and 73 time delays as may arise from the multistep nature of protein 74 expression as well as domain growth and the resulting changes 75 in source and sink terms may severely affect the existence and 76 type of Turing patterns, though some of these effects as well 77 as further regulatory interactions may somewhat increase the 78 size of the Turing space [25-32]. 
We recently noticed that ligand-receptor interactions of 31 the form shown in Fig. 1(a) can give rise to Turing patterns 82 [33-36] as long as the following constraints are met by the the 83 receptor-ligand interaction:

${ }_{84}$ (i) Ligands must diffuse much faster than receptors ${ }_{85}(d \gg 1)$, as is generally the case [37-40].

86 (ii) Receptor-dependent ligand removal must dominate 87 over receptor-independent ligand decay, as is generally the 88 case because unspecific decay is typically much slower than 89 active protein turnover.

90 (iii) Ligands and receptors must bind cooperatively, as is 91 the case for many ligand-receptor pairs [41-49].

92 (iv) Ligand-receptor complex formation must be fast com93 pared to the other processes, such that we have a quasi steady state for the ligand-receptor complex concentration. This is the case if the on-rate is very high, i.e., binding is diffusion limited, as is the case for many ligand-receptor pairs [50].

(v) The receptor-ligand complex must upregulate the receptor concentration, as has been observed for several receptor systems [51-57]. This positive feedback needs to operate far from saturation, i.e., if we describe the positive regulation by a
Hill function of the form $\frac{R^{2} L}{R^{2} L+K}$, we require $R^{2} L \ll K$. Thus, 102 this positive feedback must be rather inefficient.

If these conditions are met, the interactions between the ${ }_{104}$ receptor, $R$, and the ligand, $L$, result in Schnakenberg-type 105 kinetics [58] of the form

$$
\begin{gathered}
\frac{\partial R}{\partial t}=\Delta R+\gamma f(R, L) \text { with } f(R, L)=a-R+R^{2} L, \\
\frac{\partial L}{\partial t}=d \Delta L+\gamma g(R, L) \text { with } g(R, L)=b-R^{2} L,
\end{gathered}
$$

which correspond to the so-called activator-depleted substrate 107 Turing kinetics, first described by Gierer and Meinhardt [5], 108 and which are very similar to the chemical Turing system first 109 described by Prigogine and coworkers [7]. The detailed deriva- 110 tion of these equations for receptor-ligand interactions can be 111 found in previous publications [33-36] and in Appendix A. 112 The $\Delta R$ and $d \Delta L$ terms represent the diffusion terms, where 113 $d$ is the relative diffusion constant of ligand and receptor. ${ }_{114}$ Ligands typically diffuse faster than their receptors, $d \gg 1 \quad 115$ [37-40,59], thus naturally meeting the Turing condition of 116
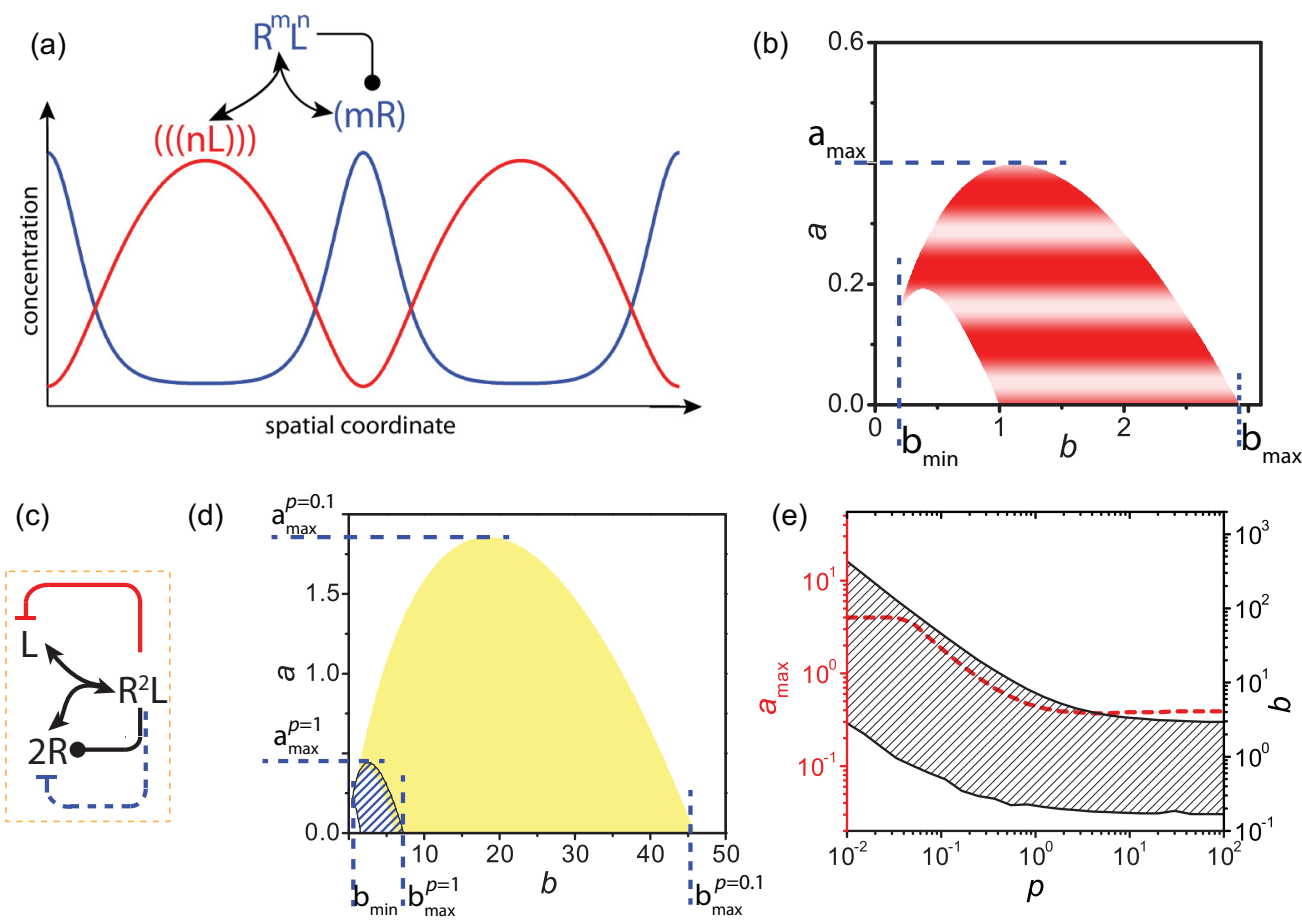

FIG. 1. (Color online) Ligand-receptor interactions can give rise to Turing patterns. (a) Spatial patterns via a Turing mechanism can result from cooperative receptor-ligand interactions, where $m$ receptors $(R)$ and $n$ ligand molecules $(L)$ form an active complex that upregulates the receptor concentration by increasing its expression, limiting its turnover or similar. Importantly, the highest receptor and ligand concentrations are observed in different places. (b) In case of the standard network [panel (a)], Turing patterns emerge only for a small subset of the parameter range of the receptor and ligand production rates, $a$ and $b . a_{\max }$ denotes the maximal value of the receptor production rate, while $b_{\min }$ and $b_{\max }$ denote the minimal and maximal ligand production rates. (c) Additional feedbacks (solid, red and dashed, blue arrows) can be mediated by the ligand-receptor complex, $R^{2} L ; \leftrightarrow$ indicates receptor-ligand interactions, $\dashv$ inhibitory interactions, and $-\bullet$ up-regulating interactions. (d) The negative feedbacks in panel (c) (network U5 in Fig. S1 [23]) result in a larger Turing space when the response threshold $p$ is lowered from $p=1$ (blue shaded area) to $p=0.1$ (solid, yellow area). (e) The size of the Turing space for the network in panel (c) (network U5 in Fig. S1 [23]) increases as the response threshold $p$ is lowered. As a measure for the size of the Turing space, we record the maximum of the receptor production rate, $a_{\max }$, and the ratio of the maximal and minimal ligand production rates $b_{\text {ratio }}=\frac{b_{\max }}{b_{\min }}$, for which Turing patterns can emerge. $a=0$ is part of the Turing space and negative values of $a$ have no physiological interpretation. 
17 different diffusivities. Receptor diffusion is restricted to single 18 cells, and we have previously shown that patterns also emerge 19 on such cellularized domains [35]. The constants $a$ and $b$ 20 are the receptor and ligand production rates. The $-R$ term 21 describes the ligand-independent decay of the receptor at a rate 122 proportional to the available receptor concentration, so-called ${ }_{23}$ linear decay. The term $R^{2} L$ represents the quasi-steady24 state concentration of the receptor-ligand complex. Signaling 125 complexes with a different stochiometry also result in Turing 126 patterns [35]. The "minus" term in Eq. (2) then reflects the 27 receptor-dependent ligand removal rate, while the "plus" term 128 in Eq. (1) reflects the combined effects of ligand-induced receptor removal and ligand-induced receptor accumulation on the cell membrane (by increased transcription, translation, recycling, less constitutive removal, or similar). The $\gamma$ term arises in the nondimensionalization of the model [Eq. (A12)] and is useful as it is proportional to the domain area, and it gives the relative strength of the reaction and diffusion terms [3].

A number of ligand-receptor systems meet the above conditions, including Hedgehog and its receptor PTCH $[34,35,47,55,56]$, BMPs and their BMP receptors [36,48,49,57], GDNF and its receptor RET [33,41,42,51,52], as well as FGFs and their FGF receptors [43-46,53,54]. Thus, all of these proteins are multimers, and, by a range of mechanisms, the formation of the multimeric ligand-receptor complexes enhances the concentration of receptors on the membrane, as recently reviewed [59]. We further showed that models based on these proteins could recapitulate the relevant wildtype and mutant expression patterns in the respective developmental systems [33-36,60].

Here we show that ligand-receptor-based Turing mechanisms can have significantly enlarged Turing spaces if we include negative feedbacks or couple several Turing modules, as generally found in biological systems. Similarly, the restriction of receptors to single cells and their clustering further increases the size of the Turing space. We conclude that a receptor-ligand-based Turing mechanism offers a realistic mechanism to implement the Turing mechanism in a biological setting. The observation that the restriction of receptors to cells is sufficient to massively increase the Turing space offers an explanation of how Turing patterns may have first evolved in nature; additional feedbacks could then further enlarge the Turing space.

\section{RESULTS}

162

The Turing mechanism has been analyzed extensively, and 163 the parameter space that permits Turing patterns to emerge 164 can easiest be determined with the help of a linear stability 165 analysis [3]; see the Appendix B. To keep the analysis feasible, 166 it is advisable to consider as models that are as simple 167 as possible and to restrict the number of parameters to a 168 minimum. The nondimensional ligand-receptor-based Turing 169 model [Eqs. (1) and (2)] has four parameters: the relative 170 ligand-receptor diffusion constant $d$, the receptor production 171 rate $a$, the ligand production rate $b$, and the scaling factor $\gamma$. 172 The parameters $a, b$, and $d$ determine whether Turing patterns 173 can emerge, while the scaling factor $\gamma$ determines whether the 174 domain is sufficiently large for Turing patterns to emerge.
We therefore do not need to analyze $\gamma$ here. The relative ${ }_{175}$ diffusion constant of ligands and receptors, $d$, affects the size 176 of the Turing space in that a larger $d$ results in a larger Turing 177 space [3]. Since this effect is well documented, but limited by 178 the physiological difference between the diffusion constants of ${ }_{179}$ ligands and receptors, we fixed the relative diffusion constant 180 in our analysis. For a simple receptor-ligand-based Turing 181 system, in which receptor and ligand bind cooperatively 182 and upregulate the receptor concentration [Fig. 1(a)], both 183 parameter values $a$ and $b$ produce Turing patterns only within 184 a small range [Fig. 1(b)], i.e., the ligand production rate can 185 at most be halved or doubled without leaving the Turing 186 space. The Turing space is thus very small, even though ${ }_{187}$ the relative diffusion constant, $d=50$, between ligands and 188 receptors was chosen to be rather large compared to what ${ }_{189}$ could be justified for two soluble ligands. We will now 190 analyze the impact of feedbacks, receptor clustering, and the 191 restriction of receptors to single cells on the size of the Turing 192 space.

\section{A. The impact of feedbacks on the Turing space of a single receptor-ligand-based Turing module}

Feedbacks are ubiquitous in biological signaling systems. 196 In the framework of receptor-ligand-based Turing mecha- 197 nisms, feedbacks result from regulatory interactions of the 198 receptor-ligand complex, $R^{2} L$ [Fig. 1(c)]. To encode feed- 199 backs mediated by receptor-ligand signaling, we modified the 200 reaction terms $f(R, L)$ and $g(R, L)$ in the Turing model [Eq. (1) 201 and (2)]. (See the Supplemental Material [23] for the list of 202 all tested models with additional feedbacks.) Thus a positive 203 feedback on receptor or ligand expression would be obtained 204 by adding a term $p R^{2} L$ to the respective equation and/or by 205 multiplying the constitutive receptor and ligand production 206 rates $a$ and $b$ with the factor $\frac{R^{2} L}{R^{2} L+p}$. A negative feedback 207 would be obtained by multiplying the constitutive receptor 208 and ligand expression rates $a$ and $b$ with the factor $\frac{1}{R^{2} L / p+1} \cdot 209$ The new parameter $p$ represents the response threshold to the 210 receptor-ligand complex. Figure 1(d) illustrates the impact of 211 feedbacks on the Turing space for the regulatory system with 212 two additional negative feedbacks shown in Fig. 1(c). For a 213 large response threshold $(p=1)$ the Turing space is similar 214 in size to the nonfeedback case [compare the blue shaded 215 area in Fig. 1(d) to the Turing space in Fig. 1(b)]. As we 216 lower the response threshold to $p=0.1$ and thus increase the 217 strength of the negative feedbacks the Turing space increases 218 in size, i.e., both the maximal receptor production rate, $a_{\max },{ }_{219}$ as well as the range of ligand expression rates $\left[b_{\min }, b_{\max }\right] 220$ increase [solid, yellow area in Fig. 1(d)]; the minimum of $a$ is 221 negative and $a_{\max }$ thus defines the size of the physiological 222 parameter range, $\left[0, a_{\max }\right]$. As the response threshold $p$ is ${ }_{223}$ lowered further, the size of the Turing space further increases 224 [Fig. 1(e)].

We next systematically analyzed 11 positive, negative, 226 and mixed feedback architectures that were obtained by 227 including feedbacks of the receptor-ligand complex $\left(R^{2} L\right)$ on ${ }_{228}$ the receptor $(a)$ and/or ligand production rates $(b)$, as well ${ }_{229}$ as on the rate of receptor up-regulation upon receptor-ligand 230 binding (for details see Appendix B, Fig. S1). Figures 2(a) 231 and 2(B) shows the three cases with the largest Turing space 232 
(a)

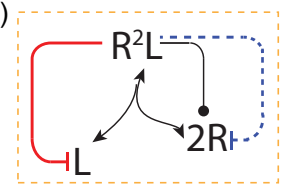

(b) $10^{2}$

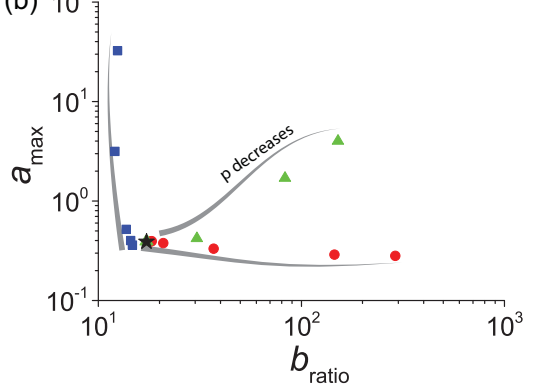

(a)

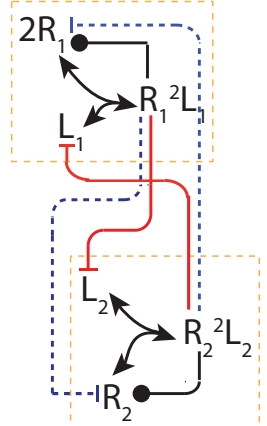

(b)

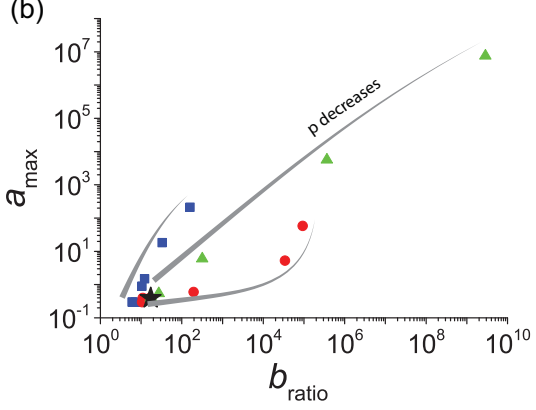

FIG. 2. (Color online) Negative feedbacks by receptor-ligand complexes result in Turing patterns with large Turing spaces. (a) The simulated network architecture. Two receptors $R$ interact with one dimeric ligand $L$ to form a receptor-ligand complex $R^{2} L$ (black arrows, $\leftrightarrow$ ). The receptor-ligand complex upregulates the presence of receptor $(-\bullet)$. In addition to these core interactions that can result in a Turing mechanism, we considered negative feedbacks $(\dashv)$ on the ligand production (red, solid arrow) and/or the receptor production (blue dashed arrow). (b) A negative feedback on the receptor production rate [blue dashed arrow in panel (a)] increases the Turing parameter space for the receptor production rate, $a$ [blue squares in panel (b)], compared to the standard network [black part of the network in panel (a) and black star in panel (b)]. A negative feedback on ligand production [red, solid arrow in panel (a)] enlarges the Turing parameter space for the ligand production rate, $b$ [red circles in (b)]. In the presence of both feedbacks the Turing parameter space is enlarged along both axes [green triangles in panel (b)]. The feedback effects are stronger the lower the feedback threshold, $p$ $(p=0.01,0.1,1,10,100)$. The gray arrow indicates the direction in which the feedback threshold, $p$, decreases.

233 of the 11 cases analyzed. For better readability, we only 234 record the maximal receptor production rate, $a_{\max }$, as well 235 as the ratio, $b_{\text {ratio }}=\frac{b_{\max }}{b_{\min }}$, of the maximal and minimal ligand 236 production rates that permit Turing patterns to emerge. We 237 note that the ratio $b_{\text {ratio }}=\frac{b_{\max }}{b_{\min }}$ is biologically more relevant 238 than the absolute size of the Turing space, $\Delta b=b_{\max }-b_{\min }$, 239 because in biology relative changes in regulatory control and 240 thus in production rates are particularly relevant; the absolute 241 values are typically very difficult to measure. The largest Turing spaces are obtained with negative feedbacks. When the negative feedback is applied to the constitutive receptor expression, $a$ (blue squares), the maximal value of $a$ increases relative to the standard model (black star) as the response threshold, $p$, is lowered; the minimum of $a$ is negative and $a_{\text {max }}$ thus defines the size of the physiological parameter range, $\left[0, a_{\max }\right]$. If a feedback is applied to the ligand expression rate, $b$, then, as the response threshold, $p$, is lowered, the range of $b$ increases (red circles) compared to the standard model (black star). The largest Turing spaces, expanded both along the $a$ and $b$ axes, are observed when negative feedbacks are applied to both the receptor and ligand expression rates (green triangles). The impact of the negative feedbacks can be observed for a wide range of the new parameters, $p$, and becomes stronger the smaller the value of the response threshold $p$ [Fig. 2(b)]. As the response threshold $p$ is increased, the maximal values of $a$, and the range of $b$, attain the value of the standard receptor-ligand model and thus all converge to the black star in Fig. 2(b). In summary, substantially enlarged Turing spaces are observed
FIG. 3. (Color online) Coupling of several receptor-ligand-based Turing modules further enlarges the Turing space. (a) The simulated network architecture. Two receptor-ligand-based Turing modules, as analyzed in Fig. 2 (black arrows, $\leftrightarrow,-\bullet$ ), are coupled via additional negative feedbacks $(-1)$ on the ligand production rates (red solid arrows) and/or the receptor production rates (blue dashed arrows). (b) A negative feedback on the receptor production rate [dashed blue line in panel (a)] increases the Turing parameter space for the receptor production rate, $a$ [blue squares in panel (b)] compared to the standard network [black part of the network in Fig. 2(a) and black star in panel (b)]. A negative feedback on ligand production [red solid arrow in panel (a)] enlarges the Turing parameter space for the ligand production rate, $b$ [red circles in (b)]. In the presence of both feedbacks the Turing parameter space is enlarged along both axes [green triangles in panel (b)]. The feedback effects are stronger the lower the feedback threshold, $p(p=0.01,0.1,1,10,100)$. The gray arrow indicates the direction in which the feedback threshold, $p$, decreases.

when signaling by the the receptor-ligand complex lowers the 261 receptor production rate [Fig. 2(b), blue], the ligand production 262 rate [Fig. 2(b), red circles], or both [Fig. 2(b), green triangles]. 263

\section{B. Coupled Turing modules}

264

In patterning processes, several receptor-ligand systems 265 often interact, e.g., SHH, FGF10, and BMP together with 266 their receptors regulate branching morphogenesis of the lung 267 and several glands, while GDNF, FGF10, and WNT and 268 their receptors regulate kidney branching morphogenesis, as 269 recently reviewed [59]. We were therefore interested in how 270 the interaction of several such Turing modules would affect 271 the Turing space.

To that end, we carried out a systematic analysis of 273 possible feedback interactions between two separate receptor- 274 ligand-based Turing systems (for details see Appendix B, 275 Sec. E). The studied network architectures, systems of 276 equations, and Turing spaces are shown in Fig. S1 [23]. 277 Figure 3(a) summarizes the coupled Turing modules with 278 the largest Turing spaces. Here, as for uncoupled modules 279 (Fig. 2), the largest Turing space is observed when a negative 280 feedback acts on the production rates (Fig. 3). We notice 281 that coupling of the two Turing systems via a negative 282 feedback on the constitutive receptor expression rates, $a$, 283 results mainly in an increase in the parameter space of $a{ }_{284}$ [Fig. 3(b), blue squares], while coupling the two Turing 285 systems via a negative feedback on the constitutive ligand 286 

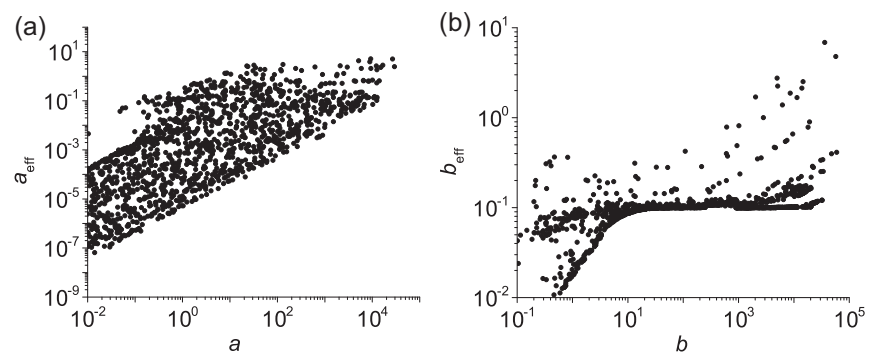

FIG. 4. Negative feedbacks enlarge the Turing space by limiting the effective production rates. The plot of the (a) effective receptor production rate $a_{\mathrm{eff}}=\frac{a}{\max \left(R^{2} L\right) / p+1}$ versus the receptor production rate $a$, and (b) the plot of the effective ligand production rate $b_{\text {eff }}=\frac{b}{\max \left(R^{2} L\right) / p+1}$ versus $b$ show that, as a result of the negative feedbacks, the effective production rates remain in a narrow range, even as $a$ and $b$ are greatly changed. The calculation was carried out for the symmetrically coupled Turing system, shown in green in Fig. 3(b).

production rate $b$ results mainly in an increase in the parameter 38 space for $b$ [Fig. 3(b), red circles]. The asymmetrically 289 coupled modules with one feedback on $a$ and one on $b$ 290 have a very large (possibly infinitely large) parameter space 291 [Fig. S1 [23], panels (C6), (C8), and (C10)]. However, the 292 parameter range is very narrow and extends towards infinity 293 only along the $b$ axis while it is bounded above on the $a$ 294 axis. A massive increase in the size of the Turing space is 295 observed when the two Turing modules are coupled by four 296 negative feedbacks, such that all constitutive receptor and 297 ligand expression rates are regulated by negative feedbacks 298 [Fig. 3(b), green triangles, and Fig. S1 [23], panel (C11)]. 299 In this case, the parameter space dramatically increases in soo both directions as $p$ is lowered, such at that already at $p=0.1$, the parameter ranges of both $a$ and $b$ expand by 02 more than four orders of magnitude compared to a single 3 receptor-ligand-based Turing model and further increase as ${ }_{304} p$ is lowered [Fig. 3(b), green triangles, and Fig. S1 [23] 305 panel $(\mathrm{C} 11)]$. 311 for the coupled Turing systems with the largest Turing space 312 [Fig. 3(b), green triangles] versus $a$ and $b$, respectively (Fig. 4). ${ }_{313}$ We find that the effective production rates are much smaller 314 than what the parameter values $a$ and $b$ would suggest and 315 almost lie within the standard small Turing space. Thus, the 16 negative feedback effectively corrects the receptor and ligand 17 production rates and thereby enables the Turing mechanism to 18 tolerate a much wider range of production rates.

\section{The restriction of receptors to single cells enlarges the Turing space}

So far, we have treated receptors in the same way as the ligand, just with a smaller diffusion coefficient. However, receptors are confined to single cells and thus cannot diffuse (a)

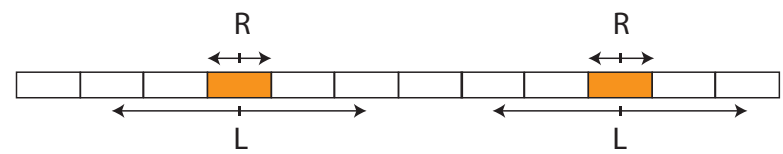

1D domain

2D domain

3D domain

(b)
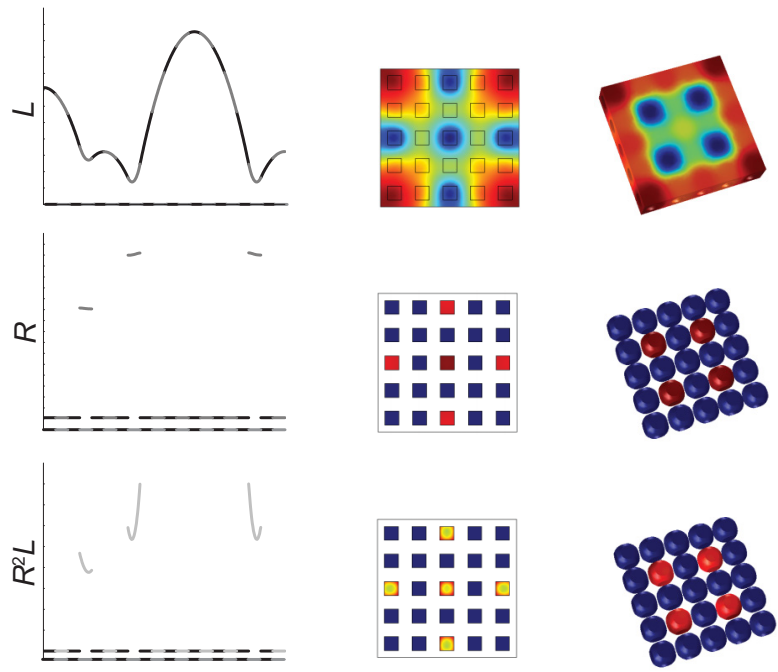

(c)

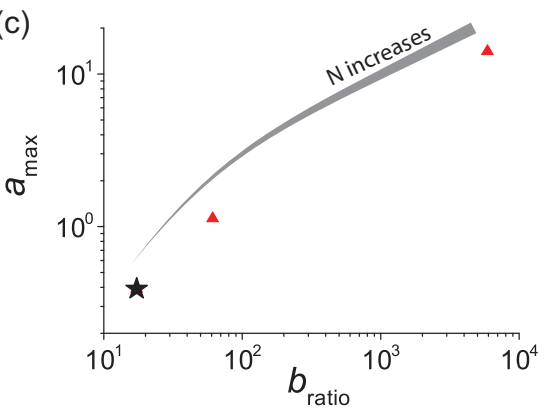

(d)

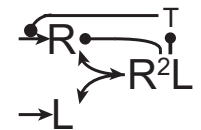

FIG. 5. (Color online) The restriction of receptors to single cells enlarges the Turing space. (a) Cartoon of the computational domain: diffusion of receptors is restricted to single cells, while ligand can diffuse over the entire computational domain. (b) Solution of the receptor-ligand model on a 1D, 2D and 3D (left to right) cellularized computational domain. The ligand (upper row), receptors (middle row), and ligand-receptor complexes (bottom row) pattern the domain. We provide the concentration levels (in arbitrary units) on the vertical axis for the 1D domain (left column), and intensities as color code (blue(dark)- low; red(light)- high) on the 2D and 3D domains. To distinguish cell boundaries on the 1D domain we alternate black and gray lines. (c) The size of the Turing space increases as the domain of fixed size is split into more cells, $N$. Triangles show the results for $N=10$ and $N=100$ cells. The black star reports the Turing space for the standard model, $N=1$. (d) Patterns of receptor-ligand complexes that extend over several cells can be obtained with a diffusive component, $T$, that is produced in response to the formation of receptor-ligand complexes, and that enhances the abundance of receptors on neighboring cells. The gray arrow indicates the direction, in which the feedback threshold, $p$, decreases.

from one cell to the next. Moreover, they often cluster on ${ }_{324}$ the cell surface. We therefore next studied Turing patterns on 325 cellular domains where receptors are confined to single cells, 326 while ligands can diffuse within the tissue [Fig. 5(a)]. The 327 
(a)

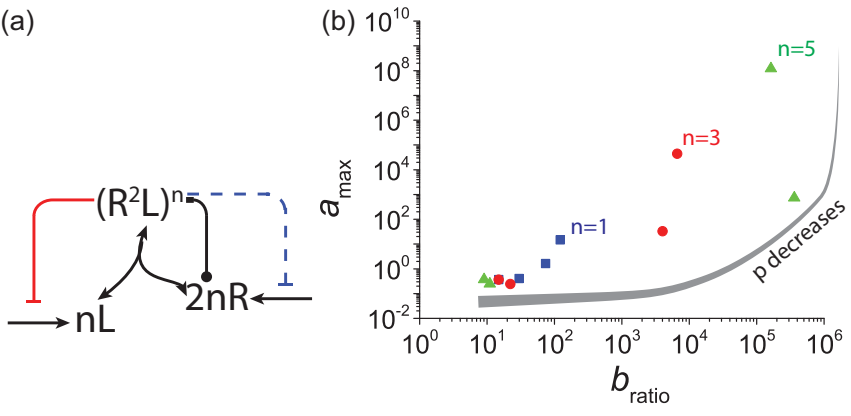

FIG. 6. (Color online) Receptor clustering enlarges the Turing space. (a) The simulated network architecture. Clusters of $2 n$ receptors $R$ interact with $n$ dimeric ligands $L$ to form a receptorligand complex $\left(R^{2} L\right)^{n}$ (black arrows, $\leftrightarrow$ ). The receptor-ligand complex upregulates the presence of receptor (black interaction, -•). In addition to these core interactions that can result in a Turing mechanism, we considered negative feedbacks on the ligand production (red solid arrow, $\dashv$ ) and/or the receptor production (blue dashed arrow, - ). (b) Higher cooperativity, $n>1$, as may result from larger receptor-ligand clusters further increases the size of the Turing space. The $n$-dependent increase was calculated for $p=0.01,0.1$, 1, 10, 100 for case U5 in Fig. S1 [23]. The gray arrow indicates the direction, in which the feedback threshold, $p$, decreases.

328 computational details of the implementation have previously 329 been described [61], and details of the implementation are 330 given in Appendix C. In brief, to restrict diffusion of receptors 331 to a single cell in one-dimensional (1D) and 2D models ${ }_{332}$ [Fig. 5(b), left and middle panels, respectively], we set no-flux 333 boundary conditions for receptor at the pseudo-cell boundary, 334 while ligand was free to diffuse in the entire domain. In the 335 3D model the cell surfaces were approximated as spheres 336 [Fig. 5(b), right panel], and both ligands and receptors were 337 produced on the spheres' surfaces. Diffusion of receptors was 338 restricted to the surface of each sphere, while ligand was free $3 з 9$ to diffuse also in the intercellular space; the details of the 340 implementation have been previously described [62].

341

We observe the emergence of patterns on 1D, 2D, and 3D 342 cellularized domains [Fig. 5(b)], and as a tissue domain of 343 a given size is divided into more (and thus smaller) cells, to 344 which the receptors are restricted, the Turing space increases 345 [Fig. 5(c)]. Interestingly, however, cells with a high level 346 of receptor-ligand complexes occur only as isolated spots 347 [Fig. 5(b), red(light) spots], while clusters of such active 348 cells are not observed. To obtain clusters of active cells we 349 have to include a second diffusively component, $T$, that is 350 secreted by the active cells and that activates neighboring 351 cells [Fig. $5(\mathrm{~d})]$.

\section{E. Receptor clustering enlarges the Turing space}

353 Receptors often cluster on cell membranes, either as 354 preclusters or induced by multimeric ligand. Clustered 355 receptor-ligand complexes may cooperate [63], such that 356 regulation is not mediated by a single ligand-receptor complex 357 but by the cluster. We then have $\left(R^{2} L / p\right)^{n}$ with $n>1$ in 358 Eq. (1) and (2) instead of $R^{2} L / p$. As we increase $n$, we observe a further increase in the size of the Turing space [Figs. 6(a) 359 and 6(b)]. In summary, both receptor clustering and the cellular 360 restriction of receptors greatly increase the Turing space. $\quad 361$

\section{F. Physiological Turing models}

362

Physiological networks harbor many feedbacks and we 363 wondered by how much the size of the Turing space would 364 be increased in physiological settings. Here we considered 365 the network that controls branching morphogenesis in the 366 lung [Fig. 7(a)]; similar networks also operate in the prostate, 367 salivary gland, and the pancreas [59]. Core to the control 368 of lung branching morphogenesis are FGF10 and SHH as 369 no branching is observed in the null mutants [64-67], and 370 expression of dominant negative $F g f r 2$ blocks lung branching 371 but not outgrowth [68].

FGF10 upregulates Shh expression [64] and the expression 373 of its own receptor, FGFR2b [53,54], while SHH signaling 374 downregulates Fgf10 expression [69] and upregulates the 375 expression of its own receptor Ptchl [70] [Fig. 7(a)]. We 376 have previously shown that the SHH-PTCH kinetics can be 377 described by Eqs. (1) and (2) [34,35]; similar equations can 378 also be derived for the FGF10-FGFR2b kinetics; see Ap- 379 pendix I for a general derivation of the ligand-receptor kinetics. 380 The particular stoichiometry in Eq. (1) and (2) assumes the 381 binding of one ligand dimer to two receptor monomers. In 382 the case of FGF10, monomeric binding of one FGF10 dimer 383 to its trivalent FGFR2b receptor triggers dimerization of the 384 FGF10-receptor complex [46]; $\mathrm{SHH}$ is a multimer that may 385 form higher-order complexes with its receptor PTCH1 [71]. 386 We have previously shown that similar Turing patterns can be ${ }_{387}$ observed also with such very different stochiometries [35]. For 388 ease of comparison, we stick to the standard model (Eq. 1-2) 389 for the FGF10 and SHH modules, though we note that larger 390 SHH-PTCH1 clusters would further increase the Turing space 391 [Figs. 5(e) and 5(F)]. The two signaling factors interact in that 392 FGF10 upregulates Shh expression [64], while SHH signaling 393 downregulates FgflO expression [69]. The equations for the 394 coupled network [Fig. 7(b)] are thus given by

$$
\begin{aligned}
\text { PTCH1: } & \dot{R}_{1}=\Delta R_{1}+f\left(R_{1}, L_{1}, R_{2}, L_{2}\right) \\
\text { SHH: } & \dot{L_{1}}=d \Delta L_{1}+g\left(R_{1}, L_{1}, R_{2}, L_{2}\right) \\
\text { FGFR2b: } & \dot{R}_{2}=\Delta R_{2}+\widetilde{f}\left(R_{1}, L_{1}, R_{2}, L_{2}\right) \\
\text { FGF10: } & \dot{L_{2}}=d \Delta L_{2}+\widetilde{g}\left(R_{1}, L_{1}, R_{2}, L_{2}\right)
\end{aligned}
$$

with

$$
\begin{aligned}
& f\left(R_{1}, L_{1}, R_{2}, L_{2}\right)=a_{1}-R_{1}+q R_{1}^{2} L_{1} \\
& g\left(R_{1}, L_{1}, R_{2}, L_{2}\right)=b_{1}-R_{1}^{2} L_{1}+p_{1} R_{2}^{2} L_{2} \\
& \widetilde{f}\left(R_{1}, L_{1}, R_{2}, L_{2}\right)=\frac{a_{2}}{1+\frac{R_{2}^{2} L_{2}}{p_{2}}}-R_{2}+q R_{2}^{2} L_{2} \\
& \widetilde{g}\left(R_{1}, L_{1}, R_{2}, L_{2}\right)=\frac{b_{2}}{1+\frac{R_{1}^{2} L_{1}}{p_{2}}}-R_{2}^{2} L_{2} .
\end{aligned}
$$

Here $R_{1}$ represents the receptor PTCH1, $L_{1}$ the ligand 397 $\mathrm{SHH}, R_{2}$ the receptor FGFR2b, and $L_{2}$ the ligand FGF10. 398 

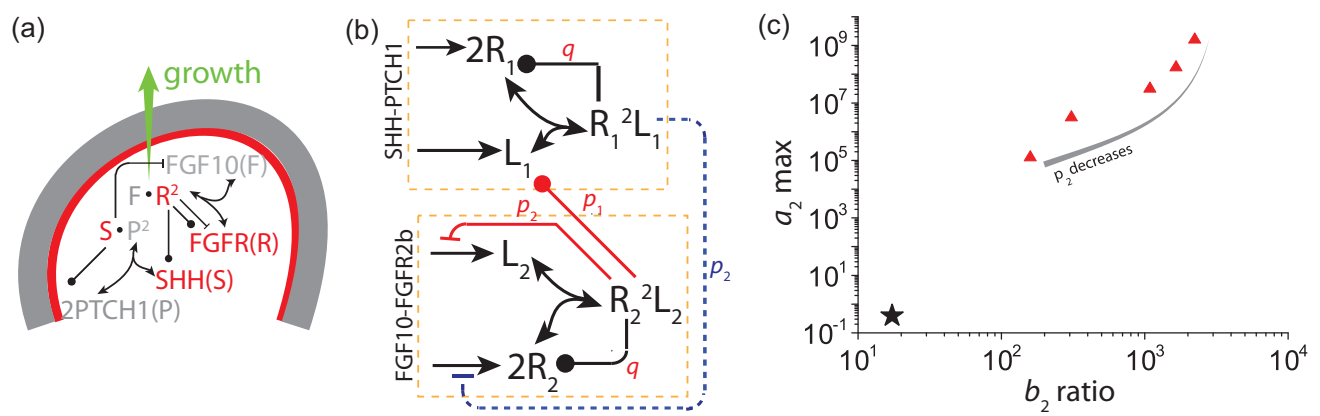

FIG. 7. (Color online) Substantially enlarged Turing spaces for physiological networks. (a) The SHH-FGF10 network in the control of lung branching morphogenesis. For details see the text. (b) Schematic representation of the regulatory network for lung branching morphogenesis in panel (a). (c) The Turing space of such a physiological model is huge and further increases as the feedback threshold, $p$, is lowered. The red triangles represent the Turing spaces for $p_{1}=q=0.1$ (positive feedback on ligand and receptor, respectively) and $p_{2}=0.01,0.1,1,10$, 100 (negative feedback); the black star represents the size of the Turing space of the standard network in Fig. 2(a) (black part). The gray arrow indicates the direction in which the feedback threshold, $p$, decreases. $\leftrightarrow$ indicates binding interactions, $\dashv$ indicates inhibitory interactions, and $-\bullet$ indicates up-regulating interactions.

399 The SHH-PTCH1 complex $R_{1}^{2} L_{1}$ upregulates the receptor ${ }_{400}$ PTCH1 [70], i.e., $+q R_{1}^{2} L_{1}$ in the term $f\left(R_{1}, L_{1}, R_{2}, L_{2}\right)$, 401 and inhibits the production of FGF10 [70], i.e., $\frac{b_{2}}{1+\frac{R_{1}^{2} L_{1}}{p_{2}}}$ in 402 the term $\widetilde{g}\left(R_{1}, L_{1}, R_{2}, L_{2}\right)$. The FGF-receptor complex, $R_{2}^{2} L_{2}$, 403 upregulates the production of SHH [64], i.e., $p_{1} R_{2}^{2} L_{2}$ in the 404 term $g\left(R_{1}, L_{1}, R_{2}, L_{2}\right)$ and both upregulates, i.e., $q R_{2}^{2} L_{2}$ in 405 term $\widetilde{f}\left(R_{1}, L_{1}, R_{2}, L_{2}\right)$, and downregulates, i.e., $\frac{a}{1+\frac{R_{2}^{2} L_{2}}{p_{2}}}$ in term $406 \tilde{f}\left(R_{1}, L_{1}, R_{2}, L_{2}\right)$, the FGF receptor FGFR2b [53,54]. The ${ }_{407}-R_{1}^{2} L_{1}$ and $-R_{2}^{2} L_{2}$ terms represent ligand removal by receptor 408 binding; receptor removal by ligand binding is absorbed 409 in the $+q R_{1}^{2} L_{1}$ and $+q R_{2}^{2} L_{2}$ terms as signaling-dependent 410 receptor upregulation dominates ligand-induced receptor 411 removal.

${ }_{412}$ We find that the combination of these two modules ${ }_{413}[$ Fig. 7(b)] increases the range of the receptor production rate, $414 a$, by about $10^{9}$-fold as the threshold $p$ is lowered to 0.01 , while 415 the relative range of the ligand production rate, $b_{2}$, increases 416 about 100-fold compared to the single receptor-ligand-based 417 Turing model [Fig. 7(c)].

\section{8 \\ III. DISCUSSION}

419 Turing mechanisms can reproduce a wide range of biolog420 ical patterning phenomena. However, it has remained unclear 421 how they may be implemented on the molecular scale and how 422 they could evolve in spite of the small sizes of their Turing ${ }_{423}$ spaces. We propose that ligand-receptor interactions give rise 424 to Turing patterns, and we show that negative feedbacks, the ${ }_{425}$ coupling of Turing modules, and the restriction of receptors to 426 single cells can greatly increase the size of the Turing space ${ }_{427}$ (Figs. 2, 3, and 5) and thus increase the range of parameter ${ }_{428}$ values for which Turing patterns will emerge in biological 429 systems.

The conditions for ligand-receptor-based Turing mecha${ }_{431}$ nisms, as summarized in the Introduction, are met by many 432 different ligand-receptor pairs, and we have previously shown that receptor-ligand-based Turing mechanisms can indeed ${ }^{433}$ well describe the patterning processes for a range of devel- ${ }_{434}$ opmental systems [33-36,59]. Equally, negative feedbacks 435 are prevalent in biological regulation and have previously ${ }_{436}$ been shown to enable robustness to noise [72] and transient ${ }_{437}$ responsiveness [73]. We now propose that negative feedbacks ${ }_{438}$ enable robust patterning also for receptor-ligand-based Turing ${ }_{439}$ mechanisms. Interestingly, also the restriction of receptors to 440 single cells can further increase the size of the Turing space ${ }_{441}$ (Fig. 5). This suggests a way that Turing mechanisms may have 442 first evolved. Cooperative interactions in receptor clusters and ${ }_{443}$ the introduction of feedbacks as well as the coupling of several ${ }_{444}$ Turing modules may then have further increased the size of ${ }_{445}$ the Turing space.

It will be important to test our theoretical insights by ${ }_{447}$ synthetically constructing such a ligand-receptor-based Turing 448 mechanism and by establishing the key parameter values 449 (rates of production, decay, diffusion coefficients, endogenous 450 concentrations, etc.) in the living systems. The Turing space ${ }^{451}$ of ligand-receptor systems with additional negative feedbacks ${ }_{452}$ should be sufficiently large that synthetic biology approaches ${ }_{453}$ can now obtain Turing patterns in spite of the difficulties ${ }^{454}$ to accurately control kinetic rates in synthetic biology ap- 455 proaches. Given their robustness and flexibility, we propose 456 that receptor-ligand-based Turing mechanisms are the likely ${ }^{457}$ standard way that Turing mechanisms are implemented in ${ }_{458}$ nature.

\section{ACKNOWLEDGMENT}

The authors thank Patrick Fried and Jannik Vollmer for ${ }^{461}$ discussions.

APPENDIX A: DERIVATION OF THE EQUATIONS FOR THE RECEPTOR-LIGAND SIGNALING MODEL

As previously derived [33-36], the dynamics of receptors, 465 $R$, ligands, $L$, and the ligand-receptor complex, $C$ [Fig. 1(c)], 466 
can be described by the following set of equations:

$$
\begin{aligned}
& {[\dot{\mathrm{L}}]=\underbrace{\bar{D}_{L} \bar{\Delta}[\mathrm{L}]}_{\text {diffusion }}+\underbrace{\bar{\rho}_{\mathrm{S}}}_{\text {production }} \underbrace{-\bar{\delta}_{L}[\mathrm{~L}]}_{\text {degradation }} \underbrace{-n \bar{k}_{\text {on }}[\mathrm{R}]^{m}[\mathrm{~L}]^{n}+n \bar{k}_{\text {off }}[\mathrm{C}]}_{\text {complex formation }},} \\
& {[\dot{\mathrm{R}}]=\underbrace{\bar{D}_{R} \bar{\Delta}[\mathrm{R}]}_{\text {diffusion }}+\underbrace{\bar{\rho}_{R}+\mu([\mathrm{C}])}_{\text {production }} \underbrace{-\bar{\delta}_{P}[\mathrm{P}]}_{\text {degradation }} \underbrace{-m k_{\mathrm{on}}[\mathrm{R}]^{m}[\mathrm{~L}]^{n}+m \bar{k}_{\mathrm{off}}[\mathrm{C}]}_{\text {complex formation }},}
\end{aligned}
$$

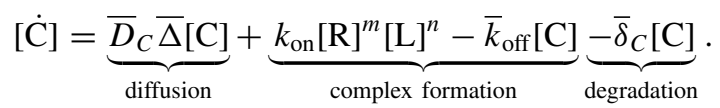

468 Here $[\mathrm{X}]$ denotes the concentration of component $X, \bar{D}_{X}$ 469 denotes the diffusion coefficient, $\bar{\rho}_{X}$ the production rate 470 constant, and $\bar{\delta}_{X}$ the first-order degradation rate constant of 471 component $X . \mu([\mathrm{C}])$ specifies a function that describes the 472 ligand-receptor dependent up regulation of receptor produc473 tion. $k_{\text {on }}$ denotes the rate constant for the formation, and $k_{\text {off }}$ 474 the rate constant for the dissociation of the ligand-receptor 475 complex. $m$ and $n$ specify the number of receptors and ligands 476 that bind in the ligand-receptor complex.

Assuming that the dynamics of the complex are fast 478 compared to those of the other components, we can introduce 479 a quasi-steady-state approximation,

$$
0=\underbrace{k_{\text {on }}[\mathrm{R}]^{m}[\mathrm{~L}]^{n}-\bar{k}_{\text {off }}[\mathrm{C}]}_{\text {complex formation }} \underbrace{-\bar{\delta}_{C}[\mathrm{C}]}_{\text {degradation }},
$$

480 and thus arrive at the quasi-steady-state concentration of 481 complex $[\mathrm{C}]_{\mathrm{SS}}$

$$
[\mathrm{C}]_{\mathrm{SS}}=\frac{\bar{k}_{\mathrm{on}}}{\bar{k}_{\mathrm{off}}+\bar{\delta}_{C}}[\mathrm{R}]^{m}[\mathrm{~L}]^{n}=\bar{\Gamma}[\mathrm{R}]^{m}[\mathrm{~L}]^{n},
$$

482 where $\bar{\Gamma}=\frac{\bar{k}_{\text {on }}}{\bar{k}_{\text {off }}+\bar{\delta}_{\mathrm{C}}}$. The concentration of bound receptor, [C], is 483 thus proportional to $[\mathrm{R}]^{m}[\mathrm{~S}]^{\mathrm{n}}$. Furthermore, assuming that the 484 rate of receptor upregulation in response to receptor-ligand 485 signaling $\mu([\mathrm{C}])=\bar{v}[\mathrm{C}]=\bar{v} \bar{\Gamma}[\mathrm{R}]^{m}[\mathrm{~L}]^{n}$ depends linearly on 486 the ligand-receptor complex concentration, $[C]$, we obtain the 487 following set of PDEs:

$$
\begin{array}{r}
{[\dot{\mathrm{L}}]=\bar{D}_{\mathrm{L}} \bar{\Delta}[\mathrm{L}]+\bar{\rho}_{\mathrm{L}}-n \bar{\delta}_{\mathrm{C}} \bar{\Gamma}[\mathrm{R}]^{m}[\mathrm{~L}]^{n}-\bar{\delta}_{\mathrm{L}}[\mathrm{L}],} \\
{[\dot{\mathrm{R}}]=\bar{D}_{\mathrm{R}} \bar{\Delta}[\mathrm{P}]+\bar{\rho}_{\mathrm{R}}+\left(\bar{v}-m \bar{\delta}_{\mathrm{C}}\right) \bar{\Gamma}[\mathrm{R}]^{m}[\mathrm{~L}]^{n}-\bar{\delta}_{\mathrm{R}}[\mathrm{R}] .}
\end{array}
$$

${ }_{488}$ We note that the linear response of the receptor production rate 489 to receptor-ligand signaling helps to increase the size of the 490 Turing space. Based on the results in Fig. S1 [23], case U6, 491 we expect that a saturation of the response for higher ligand492 receptor concentrations, as could be described by a Hill func493 tion of the form $\mu([\mathrm{C}])=H\left(\mu([\mathrm{C}], K)=H\left(\bar{\Gamma}[\mathrm{R}]^{\mathrm{n}}[\mathrm{L}]^{\mathrm{m}}, K\right)\right.$, 494 would cause a shrinking of the Turing space.

495 Equations (A6) and (A7) converge to the classical 496 Schnakenberg equations for the following conditions:

497 (a) Receptor-independent degradation of ligand is much 498 less efficient than receptor-dependent ligand degradation, as is

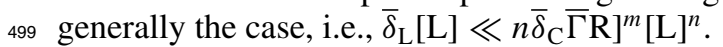

500 (b) The stochiometry of the ligand-receptor interaction 501 yields $m=2, n=1$; we note that other stochiometries also 502 yield Turing patters [35].

${ }_{503} \quad$ (c) $\bar{v}=(m+n) \bar{\delta}_{\mathrm{C}}$.

\section{Derivation of the nondimensional set of equations 504} for the receptor-ligand-based Turing mechanism

In the following, we will adopt the standard notation that 506 is used to describe Turing models, and we write $U$ for the 507 receptor concentration and $V$ for the ligand concentration; 508 $U^{m} V^{n}$ represents the quasi-steady-state concentration of the 509 receptor-ligand complex. We have previously shown that a 510 wide range of stochiometries can yield Turing patterns [35]. 511 Using $m=2, n=1$, i.e., one ligand dimer $V$ binds to two 512 monomeric receptors $U$, Eqs. (A6) and (A7) can be written as 513

$$
\begin{gathered}
\frac{\partial U}{\partial \tau}=D_{U} \Delta U+k_{1}-k_{2} U+\left(k_{5}-2 k_{3}\right) U^{2} V, \\
\frac{\partial V}{\partial \tau}=D_{V} \Delta V+k_{4}-k_{6} V-k_{3} U^{2} V,
\end{gathered}
$$

where $U=U(\tau, \mathbf{X})$ and $V=V(\tau, \mathbf{X})$ are the unknown func- 514 tions depending on the time variable $\tau$ and space variable 515 X. The coefficient $k_{1}$ then represents the constitutive re- 516 ceptor production rate, while $k_{4}$ represents the constitutive 517 ligand production rate. The term $-k_{2} U$ reflects the ligand- 518 independent receptor turnover rate while $-k_{6} V$ reflects the 519 receptor-independent ligand turnover rate. $-k_{3} U^{2} V$ reflects 520 the turnover of the receptor-ligand complex, which leads to the 521 removal of one ligand dimer, $V$, and two receptor monomers, 522 $U$. Most ligand is typically removed by this receptor- 523 dependent process, and we can therefore make the simplifying 524 approximation $k_{6}=0$. Finally, $+k_{5} U^{2} V$ reflects the signaling- 525 dependent increase in receptor emergence (which can happen 526 by a wide range of mechanisms); we will set $k_{5}=3 k_{3}$ in the ${ }_{527}$ following to recover the classical Schnakenberg equations. 528 Equations (A8) and (A9) then read

$$
\begin{gathered}
\frac{\partial U}{\partial \tau}=D_{U} \Delta U+k_{1}-k_{2} U+k_{3} U^{2} V, \\
\frac{\partial V}{\partial \tau}=D_{V} \Delta V+k_{4}-k_{3} U^{2} V .
\end{gathered}
$$

These equations can be rewritten in dimensionless form as

$$
\begin{aligned}
& \frac{\partial u}{\partial t}=\Delta u+\gamma\left(a-u+u^{2} v\right), \\
& \frac{\partial v}{\partial t}=d \Delta v+\gamma\left(b-u^{2} v\right),
\end{aligned}
$$

where

$$
\begin{aligned}
& u=U\left(\frac{k_{3}}{k_{2}}\right)^{1 / 2}, \quad v=V\left(\frac{k_{3}}{k_{2}}\right)^{1 / 2}, \quad t=\frac{D_{U} \tau}{L^{2}}, \quad \mathbf{x}=\frac{\mathbf{X}}{L}, \\
& d=\frac{D_{V}}{D_{U}}, \quad a=\frac{k_{1}}{k_{2}}\left(\frac{k_{3}}{k_{2}}\right)^{1 / 2}, \quad b=\frac{k_{4}}{k_{2}}\left(\frac{k_{3}}{k_{2}}\right)^{1 / 2}, \quad \gamma=\frac{L^{2} k_{2}}{D_{U}} .
\end{aligned}
$$


The function $u$ then represents the receptor, $v$ represents the ${ }_{533}$ ligand, and $u^{2} v$ represents the quasi-steady-state concentration ${ }_{534}$ of the receptor-ligand complex. As before, one ligand dimer ${ }_{535} v$ binds to two monomeric receptors $u$. We have previously 36 shown that also other combinations $u^{m} v^{n}$ result in Turing ${ }_{37}$ patterns [35]. The constant $\gamma a$ then represents the constitutive ${ }_{538}$ receptor production rate, while $\gamma b$ represents the constitutive 539 ligand production rate. The term $-\gamma u$ reflects the ligand540 independent receptor turnover rate, while $-\gamma u^{2} v$ reflects 541 the receptor-dependent ligand removal rate. Finally, $+\gamma u^{2} v$ 542 represents the net result of ligand-dependent receptor turnover ${ }_{543}$ and the signaling-dependent increase in receptor emergence, 544 where the latter dominates, thus the positive term.

\section{APPENDIX B: DETERMINATION OF TURING SPACES}

556 dimensionless system

$$
\begin{aligned}
& u_{t}=\gamma f(u, v)+\Delta u, \\
& v_{t}=\gamma g(u, v)+d \Delta v,
\end{aligned}
$$

557 where $t$ is the rescaled time variable, $d$ denotes (or is ${ }_{558}$ proportional to) the quotient of the diffusion coefficients $D_{U}$ 559 and $D_{V}$, and $\gamma=\mathrm{const} L^{2}$, where $L$ is a typical length scale 560 of the domain. To ensure the uniqueness of the solution we 561 endow system (B2) with initial and boundary conditions. We 562 will use homogeneous Neumann boundary condition of the 563 form

$$
\begin{aligned}
(\mathbf{n} \cdot \nabla)\left(\begin{array}{l}
u \\
v
\end{array}\right) & =0 \quad \text { on }[0, \infty) \times \partial \Omega \\
u(0, \mathbf{x}) & =u_{0}(\mathbf{x}), \quad v(0, \mathbf{x})=v_{0}(\mathbf{x}),
\end{aligned}
$$

564 because they are easy to handle and have a biological 565 interpretation (impermeable boundary). We note, however, 566 that other boundary conditions would not greatly alter the 567 following analysis. A Turing instability appears when a 568 reaction-diffusion system has a stable steady state in the 569 absence of diffusion, which loses its stability in the presence 570 of diffusion such that spatial patterns emerge.

\section{Linear stability in the absence of diffusion}

Let $u_{0}$ and $v_{0}$ denote the steady state of the diffusion-free 572 system of ordinary differential equations (ODEs)

$$
u_{t}=\gamma f(u, v), \quad v_{t}=\gamma g(u, v),
$$

and linearize the system about $\left(u_{0}, v_{0}\right)$ by introducing the 574 translated function $\mathbf{w}=\left(w_{1}, w_{2}\right)^{T}$ with $w_{1}=u-u_{0}, w_{2}=575$ $v-v_{0}$. Then the linearized system becomes

$$
\mathbf{w}_{t}=\gamma J \mathbf{w},
$$

where

$$
J=\left.\left(\begin{array}{ll}
f_{u} & f_{v} \\
g_{u} & g_{v}
\end{array}\right)\right|_{\left(u_{0}, v_{0}\right)}=\left[\begin{array}{ll}
f_{u}\left(u_{0}, v_{0}\right) & f_{v}\left(u_{0}, v_{0}\right) \\
g_{u}\left(u_{0}, v_{0}\right) & g_{v}\left(u_{0}, v_{0}\right)
\end{array}\right]
$$

is the Jacobian evaluated at the point $\left(u_{0}, v_{0}\right)$. From now on, 578 we write the partial derivatives evaluated at the steady state 579 without their arguments for brevity. The steady state of the 580 linearized system is stable, i.e., the steady state of system (B3) 581 is linearly stable if $\operatorname{Re} \lambda(J)<0$ for all eigenvalues of $J$ (see ${ }_{582}$ any textbook on ODEs), which for a two-component system ${ }_{583}$ is ensured by the conditions

$$
\operatorname{tr} J=f_{u}+g_{v}<0, \quad \operatorname{det}(J)=f_{u} g_{v}-f_{v} g_{u}>0 .
$$

\section{Diffusion-driven instability}

Now let us add diffusion to our system of ODEs and ${ }_{586}$ consider the reaction-diffusion system linearized about the ${ }_{587}$ steady state $\mathbf{w}=(0,0)^{T}$, which has the form

$$
\mathbf{w}_{t}=\gamma J \mathbf{w}+D \Delta \mathbf{w},
$$

where $D=\operatorname{diag}(1, d)$ is a diagonal matrix containing the ${ }_{589}$ diffusion coefficients of the nondimensionalized system (B2). 590 We look for a solution of the form

$$
\mathbf{w}(t, \mathbf{x})=\sum_{k} \mathbf{C}_{k} e^{\lambda_{k} t} \mathbf{W}_{k}(\mathbf{x}),
$$

where the exponents $\lambda_{k}$ determine the temporal growth of ${ }_{592}$ the solution and the time-independent functions $\mathbf{W}_{k}$ are the ${ }_{593}$ solutions of the elliptic eigenvalue problem

$$
\Delta \mathbf{W}_{k}+k^{2} \mathbf{W}_{k}=0, \quad(\mathbf{n} \cdot \nabla) \mathbf{W}_{k}=0 .
$$

For instance, in one dimension on the interval $[0, L]$ the 595 eigenvalues are $k=n \pi / L(n=0,1,2, \ldots)$, also called wave 596 numbers, and the eigenfunctions are $W(x)=\cos (n \pi x / L)={ }_{597}$ $\cos (k x)$. The constants $\mathbf{C}_{k}=\left(C_{k}^{(1)}, C_{k}^{(2)}\right)^{T}$ are the Fourier ${ }_{598}$ coefficients of the initial conditions.

Inserting Eq. (B6) into Eq. (B7) and using the fact that the 600 set of eigenfunctions of the Laplace operator $\left\{\mathbf{W}_{k}\right\}$ forms a 601 complete orthonormal system, we obtain a linearized system, 602

$$
\mathbf{w}_{t}=\gamma J \mathbf{w}+D k^{2} \mathbf{w},
$$

for each wave number $k$. Writing

$$
\operatorname{det}\left(\lambda I-\gamma J+k^{2} D\right)=0,
$$

where $I=I_{2}$ is the $2 \times 2$ identity matrix, we obtain the 604 eigenvalues $\lambda=\lambda_{k}$ of the matrix $M=\gamma J-k^{2} D$. Expanding 605 the above determinant, we obtain that $\lambda_{k}$ is the root of the ${ }_{606}$ 
607 second-order polynomial equation

$$
\begin{aligned}
& \lambda^{2}+\lambda\left[k^{2}(1+d)-\gamma\left(f_{u}+g_{v}\right)\right]+d k^{4}-\gamma\left(d f_{u}+g_{v}\right) k^{2} \\
& \quad+\gamma^{2}\left(f_{u} g_{v}-f_{v} g_{u}\right)=0 .
\end{aligned}
$$

608 Since we look for unstable solutions, we require that $\operatorname{Re} \lambda_{k}>0$ 609 for some $k \neq 0$. This means that either the coefficient of $\lambda$ 610 and/or the constant term must be negative. Since the steady 611 state is required to be linearly stable in the absence of 612 diffusion (which corresponds to the case $k=0$ ), we must have ${ }_{613} k^{2}(1+d)-\gamma\left(f_{u}+g_{v}\right)>0$. Hence, to obtain a $\lambda$ with a 614 positive real part in the presence of diffusion we require

$$
h\left(k^{2}\right):=d k^{4}-\gamma\left(d f_{u}+g_{v}\right) k^{2}+\gamma^{2}\left(f_{u} g_{v}-f_{v} g_{u}\right)<0
$$

615 for some nonzero wave number $k$. Since we require $f_{u} g_{v}-$ ${ }_{616} f_{v} g_{u}>0$ for linear stability in the absence of diffusion $617(k=0)(\mathrm{B} 4)$, it follows that $d f_{u}+g_{v}>0$ must hold. This 618 condition is not sufficient to ensure the negativity of the 619 function $h$; an elementary calculation shows that the minimum 620 of $h$ is attained at the point

$$
k_{m}^{2}=\gamma \frac{d f_{u}+g_{v}}{2 d},
$$

621 and the minimum value of $h$ is

$$
h_{\min }=h\left(k_{\mathrm{m}}^{2}\right)=\gamma^{2}\left[\left(f_{u} g_{v}-f_{v} g_{u}\right)-\frac{\left(d f_{u}+g_{v}\right)^{2}}{4 d}\right],
$$

622 which is negative if the expression in the bracket is negative. 623 In summary, the well-known conditions (see Ref. [78], 624 Sec. 2.3) for which a reaction-diffusion system with two 625 species exhibits a Turing instability are as follows:

$$
\begin{aligned}
f_{u}+g_{v}<0, & f_{u} g_{v}-f_{v} g_{u}>0, \\
d f_{u}+g_{v}>0, & \left(d f_{u}+g_{v}\right)^{2}-4 d\left(f_{u}+g_{v}-f_{v} g_{u}\right)>0,
\end{aligned}
$$

626 where all partial derivatives are evaluated at the steady state ${ }_{627}\left(u_{0}, v_{0}\right)$. We note that it is possible that these conditions are 628 satisfied but that no pattern emerges. This is the case when $h$ is 629 not negative for any $k$ within the discrete set of wave numbers 630 and only takes a negative value between two of these discrete 631 wave numbers. The distance between wave numbers shrinks as $632 \gamma$ is increased, and in the limit of infinite $\gamma$ the spectrum of $k$ is ${ }_{633}$ continuous. Since $\gamma$ is related to the size of the spatial domain, 634 it follows that on small domains pattern formation may not 635 happen, while on a sufficiently increased domain patterns may 636 be observed.

\section{4. Turing instability in interacting systems}

638 We now consider two identical reaction-diffusion systems, 639 which we couple with each other in several ways. When 640 the couplings are of the same type (i.e., when the first 641 two-component Turing system based on $u$ and $v$ is coupled 642 with the second Turing system that is based on $\widetilde{u}$ and $\widetilde{v}$ via the 643 same functions $f$ and $g$ ), then we can derive exact conditions 644 for the Turing instability, as an extension of the classical 645 results that were presented in Sec. IV B (see Ref. [3], Sec. 2.3, 646 for more details). For this let us consider systems of the form

$$
\begin{aligned}
u_{t} & =\gamma f(u, v, \widetilde{u}, \widetilde{v})+\Delta u \\
v_{t} & =\gamma g(u, v, \widetilde{u}, \widetilde{v})+d \Delta v \\
\tilde{u}_{t} & =\gamma f(\widetilde{u}, \widetilde{v}, u, v)+\Delta \widetilde{u} \\
\widetilde{v}_{t} & =\gamma g(\widetilde{u}, \widetilde{v}, u, v)+d \Delta \widetilde{v},
\end{aligned}
$$

where the functions $f$ and $g$ describe the chemical reactions, 648 $\gamma>0$ is a constant depending on the size of the domain, and ${ }_{649}$ $d>0$ is a diffusion parameter. Let $\left(u_{0}, v_{0}, \widetilde{u}_{0}, \widetilde{v}_{0}\right)$ denote the 650 steady state (assuming that there is only one or at least they are 651 isolated) of this system in the absence of diffusion (note that 652 due to the symmetry $u_{0}=\widetilde{u}_{0}$ and $v_{0}=\widetilde{v}_{0}$ ) and-just as in the ${ }_{653}$ uncoupled case (B3) - linearize the system about the steady 654 state. The linearized system has the form

$$
\mathbf{w}_{t}=\gamma J \mathbf{w},
$$

where

$$
J=\left(\begin{array}{llll}
f_{u} & f_{v} & f_{\widetilde{u}} & f_{\widetilde{v}} \\
g_{u} & g_{v} & g_{\widetilde{u}} & g_{\widetilde{v}} \\
f_{\widetilde{u}} & f_{\widetilde{v}} & f_{u} & f_{v} \\
g_{\widetilde{u}} & g_{\widetilde{v}} & g_{u} & g_{v}
\end{array}\right)
$$

is the Jacobian matrix. Note the symmetry in $J$ that arises for 657 this particular coupling. In this linearized system the steady 658 state is stable if $\operatorname{Re} \lambda(J)<0$ for all eigenvalues of $J$. The 659 eigenvalues are the roots of the characteristic polynomial $k_{J}(\lambda) \quad 660$ of $J$, which is now a fourth-order polynomial for the coupled 661 system. Due to the very special form of the coupling and the 662 resulting symmetries in $J$, the polynomial $k_{J}$ can be factorized 663 as

$$
\begin{aligned}
k_{J}(\lambda)= & {\left[\lambda^{2}+\lambda\left(-f_{u}-g_{v}-f_{\widetilde{u}}-g_{\widetilde{v}}\right)+f_{u} g_{v}-f_{v} g_{u}\right.} \\
& \left.+f_{u} g_{\widetilde{v}}-f_{\widetilde{v}} g_{u}+f_{\widetilde{u}} g_{v}-f_{v} g_{\widetilde{u}}+f_{\widetilde{u}} g_{\widetilde{v}}-f_{\widetilde{v}} g_{\widetilde{u}}\right] \\
& \times\left[\lambda^{2}+\lambda\left(-f_{u}-g_{v}+f_{\widetilde{u}}+g_{\widetilde{v}}\right)+f_{u} g_{v}-f_{v} g_{u}\right. \\
& \left.-f_{u} g_{\widetilde{v}}+f_{\widetilde{v}} g_{u}-f_{\widetilde{u}} g_{v}+f_{v} g_{\widetilde{u}}+f_{\widetilde{u}} g_{\widetilde{v}}-f_{\widetilde{v}} g_{\widetilde{u}}\right] .
\end{aligned}
$$

Hence $\operatorname{Re} \lambda(J)<0$ holds for all four eigenvalues of $J$, that is, 665 the steady state of (B9) is linearly stable if both of the factors 666 in (B10) have only roots with negative real part, i.e.,

$$
\begin{aligned}
f_{u}+g_{v}< & \pm\left(f_{\widetilde{u}}+g_{\widetilde{v}}\right), \\
f_{u} g_{v}-f_{v} g_{u}+f_{\widetilde{u}} g_{\widetilde{v}}-f_{\widetilde{v}} g_{\widetilde{u}}> & \pm\left(f_{u} g_{\widetilde{v}}-f_{\widetilde{v}} g_{u}\right. \\
& \left.+f_{\widetilde{u}} g_{v}-f_{v} g_{\widetilde{u}}\right) .
\end{aligned}
$$

Following the course of the uncoupled case, by adding 668 diffusion, we again arrive at Eq. (B7), now with the diffusion 669 matrix $D=\operatorname{diag}(1, d, 1, d)$. As before, we look for a solution of 670 the form of Eq. (B6). To this end, we determine the eigenvalues 671 $\lambda=\lambda_{k}$ for $M=\gamma J-k^{2} D$. The characteristic polynomial of 672 this matrix-given the special forms of $J$ and $D$-can be 673 factorized as the product of two second-order polynomials as 674 
675 follows:

$$
\begin{aligned}
k_{M}(\lambda)= & {\left[\lambda^{2}+\lambda\left(k^{2}(1+d)-\gamma\left(f_{u}+g_{v}+f_{\widetilde{u}}+g_{\widetilde{v}}\right)\right)+d k^{4}-\gamma k^{2}\left(d f_{u}+g_{v}+d f_{\widetilde{u}}+g_{\widetilde{v}}\right)\right.} \\
& \left.+\gamma^{2}\left(f_{u} g_{v}-f_{v} g_{u}+f_{u} g_{\widetilde{v}}-f_{\widetilde{v}} g_{u}+f_{\widetilde{u}} g_{v}-f_{v} g_{\widetilde{u}}+f_{\widetilde{u}} g_{\widetilde{v}}-f_{\widetilde{v}} g_{\widetilde{u}}\right)\right] \\
& \times\left[\lambda^{2}+\lambda\left(k^{2}(1+d)-\gamma\left(f_{u}+g_{v}-f_{\widetilde{u}}-g_{\widetilde{v}}\right)\right)+d k^{4}-\gamma k^{2}\left(d f_{u}+g_{v}-d f_{\widetilde{u}}-g_{\widetilde{v}}\right)\right. \\
& \left.+\gamma^{2}\left(f_{u} g_{v}-f_{v} g_{u}-f_{u} g_{\widetilde{v}}+f_{\widetilde{v}} g_{u}-f_{\widetilde{u}} g_{v}+f_{v} g_{\widetilde{u}}+f_{\widetilde{u}} g_{\widetilde{v}}-f_{\widetilde{v}} g_{\widetilde{u}}\right)\right] .
\end{aligned}
$$

676 To obtain a Turing instability, at least one of the roots of $k_{M}$ has to have a positive real part for some $k \neq 0$, i.e., one of the 677 factors of $k_{M}$ must have a root with $\operatorname{Re} \lambda(M)>0$. The first factor of (B12) has a root with positive real part if the coefficient 678 of $\lambda$ is negative or the constant term is negative. But since the steady state is stable in the absence of diffusion [linear stability 679 conditions (B11)] the coefficient of $\lambda$ is always positive, i.e., $k^{2}(1+d)-\gamma\left(f_{u}+g_{v}+f_{\widetilde{u}}+g_{\widetilde{v}}\right)>0$. Hence we require that

$$
h^{(1)}\left(k^{2}\right):=d k^{4}-\gamma k^{2}\left(d f_{u}+g_{v}+d f_{\widetilde{u}}+g_{\widetilde{v}}\right)+\gamma^{2}\left(f_{u} g_{v}-f_{v} g_{u}+f_{u} g_{\widetilde{v}}-f_{\widetilde{v}} g_{u}+f_{\widetilde{u}} g_{v}-f_{v} g_{\widetilde{u}}+f_{\widetilde{u}} g_{\widetilde{v}}-f_{\widetilde{v}} g_{\widetilde{u}}\right)<0
$$

680 holds for some wave number $k \neq 0$. Since we know from the linear stability conditions (B11) that the constant term is positive, 681 i.e., $f_{u} g_{v}-f_{v} g_{u}+f_{u} g_{\widetilde{v}}-f_{\widetilde{v}} g_{u}+f_{\widetilde{u}} g_{v}-f_{v} g_{\widetilde{u}}+f_{\widetilde{u}} g_{\widetilde{v}}-f_{\widetilde{v}} g_{\widetilde{u}}>0$, it follows that $d f_{u}+g_{v}+d f_{\widetilde{u}}+g_{\widetilde{v}}>0$ must hold. We 682 further need to ensure that the function $h^{(1)}$ attains a negative value for some of the wave numbers. The minimum of $h^{(1)}$ is 683 attained at

$$
k_{1, \mathrm{~m}}^{2}=\gamma \frac{d f_{u}+g_{v}+d f_{\widetilde{u}}+g_{\widetilde{v}}}{2 d}
$$

684 and the minimum value of $h^{(1)}$ is

$$
h_{\min }^{(1)}=h^{(1)}\left(k_{1, \mathrm{~m}}^{2}\right)=\gamma^{2}\left[\left(f_{u} g_{v}-f_{v} g_{u}+f_{u} g_{\widetilde{v}}-f_{\widetilde{v}} g_{u}+f_{\widetilde{u}} g_{v}-f_{v} g_{\widetilde{u}}+f_{\widetilde{u}} g_{\widetilde{v}}-f_{\widetilde{v}} g_{\widetilde{u}}\right)-\frac{\left(d f_{u}+g_{v}+d f_{\widetilde{u}}+g_{\widetilde{v}}\right)^{2}}{4 d}\right] .
$$

685 The minimum value of $h^{(1)}$ is thus negative if the expression in the bracket is negative. If the first factor of (B12) does not have roots 686 with positive real part, the second factor has to have at least one root with positive real part to obtain a Turing instability. By similar 687 reasoning as before we know from (B11) that the coefficient of $\lambda$ is again always positive: $k^{2}(1+d)-\gamma\left(f_{u}+g_{v}-f_{\widetilde{u}}-g_{\widetilde{v}}\right)>0$. 688 Hence, it is required that

$$
h^{(2)}\left(k^{2}\right):=d k^{4}-\gamma k^{2}\left(d f_{u}+g_{v}-d f_{\widetilde{u}}-g_{\widetilde{v}}\right)+\gamma^{2}\left(f_{u} g_{v}-f_{v} g_{u}-f_{u} g_{\widetilde{v}}+f_{\widetilde{v}} g_{u}-f_{\widetilde{u}} g_{v}+f_{v} g_{\widetilde{u}}+f_{\widetilde{u}} g_{\widetilde{v}}-f_{\widetilde{v}} g_{\widetilde{u}}\right)<0
$$

689 holds for some $k \neq 0$. A necessary condition for this is $d f_{u}+g_{v}-d f_{\widetilde{u}}-g_{\widetilde{v}}>0$, since the constant term in $h^{(2)}$ is positive again 690 by (B11). To obtain a sufficient condition we have to calculate the minimum of $h^{(2)}$ as before, i.e.,

$$
k_{2, \mathrm{~m}}^{2}=\gamma \frac{d f_{u}+g_{v}-d f_{\widetilde{u}}-g_{\widetilde{v}}}{2 d} .
$$

691 The minimum value of $h^{(2)}$ is

$$
h_{\min }^{(2)}=h^{(2)}\left(k_{2, \mathrm{~m}}^{2}\right)=\gamma^{2}\left[\left(f_{u} g_{v}-f_{v} g_{u}-f_{u} g_{\widetilde{v}}+f_{\widetilde{v}} g_{u}-f_{\widetilde{u}} g_{v}+f_{v} g_{\widetilde{u}}+f_{\widetilde{u}} g_{\widetilde{v}}-f_{\widetilde{v}} g_{\widetilde{u}}\right)-\frac{\left(d f_{u}+g_{v}-d f_{\widetilde{u}}-g_{\widetilde{v}}\right)^{2}}{4 d}\right] .
$$

692 In summary, the steady state has to be linearly stable if no diffusion is present, which means that all roots of (B10) have 693 negative real part, but instability appears when diffusion is added, which means that the polynomial in (B12) has to have at least 694 one root with a positive real part. Hence for Turing instability in the coupled system (B9) one of the following sets of conditions 695 has to be satisfied [(B13a) or (B13b)]:

$$
\begin{aligned}
& f_{u}+g_{v}< \pm\left(f_{\widetilde{u}}+g_{\widetilde{v}}\right), \quad f_{u} g_{v}-f_{v} g_{u}+f_{\widetilde{u}} g_{\widetilde{v}}-f_{\widetilde{v}} g_{\widetilde{u}}> \pm\left(f_{u} g_{\widetilde{v}}-f_{\widetilde{v}} g_{u}+f_{\widetilde{u}} g_{v}-f_{v} g_{\widetilde{u}}\right), \quad d f_{u}+g_{v}+d f_{\widetilde{u}}+g_{\widetilde{v}}>0, \\
& \left(d f_{u}+g_{v}+d f_{\widetilde{u}}+g_{\widetilde{v}}\right)^{2}-4 d\left(f_{u} g_{v}-f_{v} g_{u}+f_{u} g_{\widetilde{v}}-f_{\widetilde{v}} g_{u}+f_{\widetilde{u}} g_{v}-f_{v} g_{\widetilde{u}}+f_{\widetilde{u}} g_{\widetilde{v}}-f_{\widetilde{v}} g_{\widetilde{u}}\right)>0 ; \\
& f_{u}+g_{v}< \pm\left(f_{\widetilde{u}}+g_{\widetilde{v}}\right), \quad f_{u} g_{v}-f_{v} g_{u}+f_{\widetilde{u}} g_{\widetilde{v}}-f_{\widetilde{v}} g_{\widetilde{u}}> \pm\left(f_{u} g_{\widetilde{v}}-f_{\widetilde{v}} g_{u}+f_{\widetilde{u}} g_{v}-f_{v} g_{\widetilde{u}}\right), \quad d f_{u}+g_{v}-d f_{\widetilde{u}}-g_{\widetilde{v}}>0, \\
& \left(d f_{u}+g_{v}-d f_{\widetilde{u}}-g_{\widetilde{v}}\right)^{2}-4 d\left(f_{u} g_{v}-f_{v} g_{u}-f_{u} g_{\widetilde{v}}+f_{\widetilde{v}} g_{u}-f_{\widetilde{u}} g_{v}+f_{v} g_{\widetilde{u}}+f_{\widetilde{u}} g_{\widetilde{v}}-f_{\widetilde{v}} g_{\widetilde{u}}\right)>0,
\end{aligned}
$$

696 where the first line comes from the linear stability condition (hence they are the same in both cases) and the other two lines are 697 derived from the diffusion-driven instability conditions.

\section{APPENDIX C: CELLULAR MODELS}

Here we present the details of the implementation of the cellular models presented in Fig. 5. We consider 1D, 2D, and
3D cellular models. In all cases we solved Eqs. (1) and (2) but 701 with some terms restricted to certain subdomains as specified 702 below. All equations were solved on the same mesh.

\section{2}


705 We use a 1D domain, comprising $N$ subdomains of 706 equal length [Fig. 5(b)]. On every subdomain the set of 707 Eqs. (1) and (2) is solved. Ligand $L$ can diffuse freely in 708 the entire domain, while receptor $R$ is restricted to each 709 subdomain by no-flux boundary conditions. Ligand exchange 710 between subdomains is obtained by enforcing continuous 711 ligand profiles across the borders of the subdomains, i.e., 712 by requiring that the ligand value $L$ on the right-hand side 713 boundary of subdomain $i$ is the same as the ligand value $L$ on 714 the left-hand side boundary of subdomain $i+1$.

\section{2. 2D cellular models}

We use a $2 \mathrm{D}$ square domain, containing $N \times N$ equal-sized 717 subdomains of square shape. The subdomains neither intersect 718 nor overlap [Fig. 5(b)]. The following set of PDEs is defined 719 on this $2 \mathrm{D}$ domain as follows:

$$
\begin{gathered}
\frac{\partial R}{\partial t}=\Delta R+\gamma\left(a-R+R^{2} L\right) \text { on } C, \\
\frac{\partial L}{\partial t}=d \Delta L+\gamma\left\{\begin{array}{ll}
\left(b-R^{2} L\right) & \text { on } C \\
0 & \text { on } E C
\end{array},\right.
\end{gathered}
$$

720 where $C$ represents the $N \times N$ array of rectangular cellular 721 subdomains and EC refers to the rest of the 2D domain, 722 representing the extracellular space.

\section{$723 \quad$ 3. 3D cellular models}

${ }_{724}$ We use a 3D domain [Fig. 5(b)], containing $N \times N \times 1$ 725 nonoverlapping spheres that are embedded into a cuboid.

726 The following set of PDEs describes the ligand and re727 ceptor dynamics on the surface of the spheres, referred to as $C$,

$$
\begin{gathered}
\frac{\partial R}{\partial t}=\Delta R+\gamma\left(a-R+R^{2} L\right) \text { on } C, \\
\frac{\partial L}{\partial t}=d \Delta L+\gamma\left(b-R^{2} L\right) \text { on } C .
\end{gathered}
$$

Additionally, the ligand is free to diffuse in the bulk of the 729 cuboid, referred to as $E C$,

$$
\frac{\partial L}{\partial t}=d \Delta L \text { on } E C .
$$

The concentration of the ligand on the surface of the spheres 731 and in the bulk of the cuboid is linked via

$$
d \vec{n} \cdot \nabla L=\gamma\left(b-R^{2} L\right),
$$

where $\vec{n}$ is the outward normal vector. The volume inside the 733 spheres (i.e., the cell interior) is not included in the simulations 734 because we do not consider ligand or receptor internalization. 735

\section{APPENDIX D: NUMERICAL SOLUTION OF PDES WITH COMSOL}

The partial differential equations were solved in COMSOL 738 MULTIPHYSICS 4.X as described previously [61,62,74]. COMSOL 739 MULTIPHYSICS has previously been used to accurately solve a 740 variety of reaction-diffusion equations which originate from 741 chemical, biological, and engineering applications [33-36,75- 742 80]. In the following we present two tests for the numerical 743 accuracy of the solution of Turing type models obtained with 744 COMSOL MULTIPHYSICS.

\section{Accuracy of the Turing space}

746

We first test whether we obtain the same Turing space 747 numerically and analytically. To this end, we use Eq. (B8) 748 as analytical condition for a Turing instability for the Turing 749

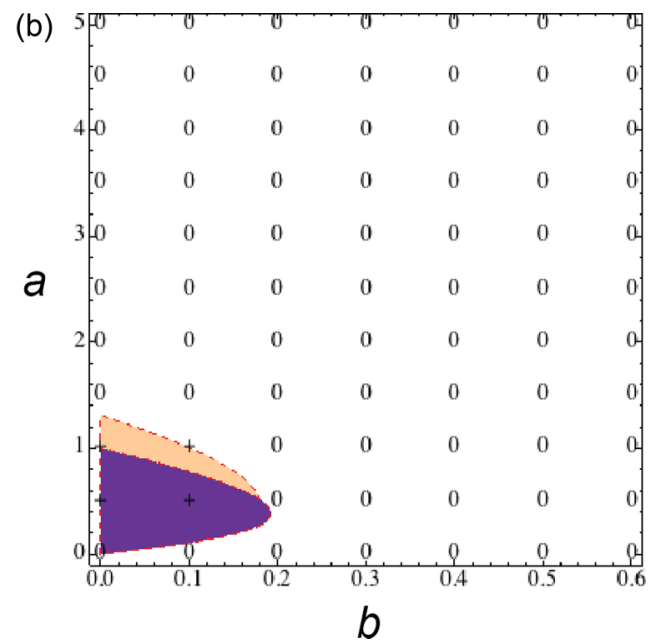

FIG. 8. (Color online) Comparison of the Turing spaces calculated numerically and those derived analytically. [(a) and (b)] The shaded regions of the parameter space indicates the area where the linear stability analysis identifies a Turing instability (yellow, light shading) or other instabilities (navy, dark shading) for Eqs. (1) and (2) with zero-flux boundary conditions. The symbols indicate the points in the parameter space where the numerical solution of Eqs. (1) and (2) with zero-flux boundary conditions yielded either pattern formation $(+)$ or not $(0)$. $\gamma$ was chosen sufficiently large that Turing patterns could emerge on the 1D domain. Panels (a) and (b) differ in the relative diffusion coefficient $d$, with (a) $d=100$ and (b) $d=10$. 
750 model given by Eqs (1) and (2). To estimate the size of the 751 Turing space numerically, we solve Eqs. (1) and (2) with 752 COMSOL. Figure 8 shows that the numerical solution of Eqs. (1) 753 and (2) in COMSOL yields pattern ( + symbols) in the part of 754 the parameter space where the analytical criterion specifies 755 either the classical Turing space (yellow region) or an unstable 756 steady state both in the presence and absence of diffusion (blue 757 region).

\section{$758 \quad 2$. Convergence of numerical solution}

759 Here we show that the numerical solution of a ligand760 receptor-based Turing model on a domain comprising two 761 layers converges with respect to the mesh size. We consider 762 the model

$$
\begin{aligned}
& \frac{\partial R}{\partial t}=\Delta R+\gamma\left(a-R+R^{2} L\right) \text { on } T_{1}, \\
& \frac{\partial L}{\partial t}=d \Delta L+\gamma\left\{\begin{array}{ll}
\left(-R^{2} L\right) & \text { on } T_{1} \\
b & \text { on } T_{2}
\end{array},\right.
\end{aligned}
$$

763 where $T_{1}$ and $T_{2}$ indicate two different tissue layers. 764 Figure 9(a) shows the calculated distribution of the receptor765 ligand complex $\left(R^{2} L\right)$; similar patterns were obtained for a 766 range of finite-element meshes with the maximum size of 767 the mesh size in the range from 0.01 to 0.1 . Figure 9(b)
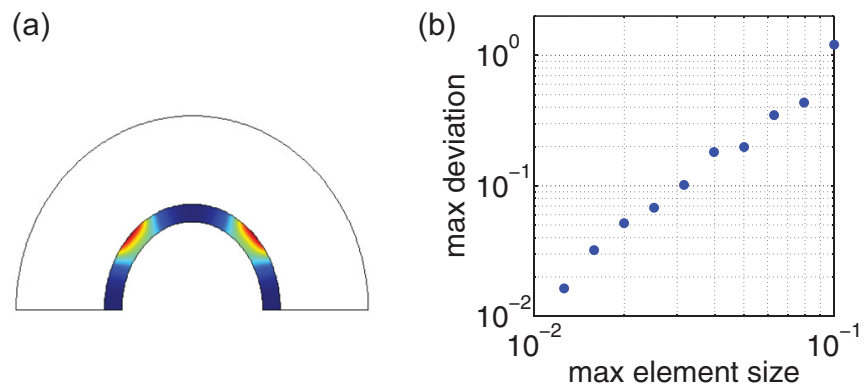

FIG. 9. (Color online) Convergence of the numerical solution. (a) Typical pattern of receptor-ligand complexes $\left(R^{2} L\right)$ on a domain comprising two subdomains. Ligand is produced in the upper domain but free to diffuse on the entire domains. Receptor is produced in the lower domain and its diffusion is restricted to the lower domain. (b) The maximum deviation of the receptor-ligand complex $\left(R^{2} L\right)$ as computed with an FEM mesh with element size equal to 0.01 from that computed at other mesh sizes.

shows that the maximum deviation in the solution decreases 768 quadratically with respect to the maximum mesh size or, 769 equivalently, decreases linearly with respect to the maximum 770 mesh edge, as expected for finite element method (FEM) with 771 first-order Lagrange elements. These tests support the previous 772 observations by others that COMSOL MULTYPHYSICS can solve 773 Turing-type equations accurately.
[1] A. M. Turing, Phil. Trans. Roy. Soc. Lond. B 237, 37 (1952).

[2] S. Kondo and T. Miura, Science 329, 1616 (2010).

[3] J. D. Murray, Mathematical Biology: II. Spatial Models and Biomedical Applications (Springer, Berlin, 2003).

[4] V. K. Vanag and I. R. Epstein, Phys. Rev. Lett. 87, 228301 (2001).

[5] A. Gierer and H. Meinhardt, Kybernetik 12, 30 (1972).

[6] I. Prigogine, J. Chem. Phys. 46, 3542 (1967).

[7] I. Prigogine and R. Lefever, J. Chem. Phys. 48, 1695 (1968).

[8] S. W. Cho, S. Kwak, T. E. Woolley, M. J. Lee, E. J. Kim et al., Development 138, 1807 (2011).

[9] A. D. Economou, A. Ohazama, T. Porntaveetus, P. T. Sharpe, S. Kondo et al., Nat. Genet. 44, 348 (2012).

[10] S. Sick, S. Reinker, J. Timmer, and T. Schlake, Science 314, 1447 (2006).

[11] J. Horvth, I. Szalai, and P. De Kepper, Science 324, 772 (2009).

[12] P. Müller, K. W. Rogers, B. M. Jordan, J. S. Lee, D. Robson et al., Science 336, 721 (2012).

[13] J. Hsia, W. J. Holtz, D. C. Huang, M. Arcak, and M. M. Maharbiz, PLoS Comput. Biol. 8, e1002331 (2012).

[14] A. Nakamasu, G. Takahashi, A. Kanbe, and S. Kondo, Proc. Natl. Acad. Sci. USA 106, 8429 (2009).

[15] S. Payne, B. Li, Y. Cao, D. Schaeffer, M. D. Ryser, and L. You, Mol. Syst. Biol. 9, 697 (2013).

[16] P. Y. Pang and M. Wang, J. Differ. Eqs. 200, 245 (2004).

[17] A. E. Hamidi, M. Garbey, and N. Ali, Nonlin. Anal. Real World Appl. 13, 1306 (2012).

[18] T. Butler and N. Goldenfeld, Phys. Rev. E 84, 011112 (2011).

[19] L. Ridolfi, C. Camporeale, P. D'Odorico, and F. Laio, Europhys. Lett. 95, 18003 (2011).
[20] D. Fanelli, C. Cianci, and F. Di Patti, Eur. Phys. J. B 86, 142 (2013).

[21] A. Madzvamuse, H. S. Ndakwo, and R. Barreira, J. Math. Biol. (2014), doi:10.1007/s00285-014-0779-6.

[22] D. Bullara, Y. De Decker, and R. Lefever, Phys. Rev. E 87, 062923 (2013)

[23] See Supplemental Material at http://link.aps.org/supplemental/ PhysRevE.xx.xxxxxx for a list of all tested models with additional feedbacks.

[24] J. Murray, J. Theor. Biol. 98, 143 (1982).

[25] E. Crampin, E. Gaffney, and P. Maini, Bull. Math. Biol. 61, 1093 (1999).

[26] J. Gjorgjieva and J. Jacobsen, Discrete Contin. Dyn. System, Special Issue 2007, 436 (2007), http://aimsciences.org/journals/ displayArticles.jsp?paperID $=2850$.

[27] A. Madzvamuse, E. A. Gaffney, and P. K. Maini, J. Math. Biol. 61, 133 (2010)

[28] T. E. Woolley, R. E. Baker, E. A. Gaffney, and P. K. Maini, Phys. Rev. E 84, 046216 (2011).

[29] P. K. Maini, T. E. Woolley, R. E. Baker, E. A. Gaffney, and S. S. Lee, Roy. Soc. Interface Focus 2, 487 (2012).

[30] T. E. Woolley, R. E. Baker, E. A. Gaffney, P. K. Maini, and S. Seirin-Lee, Phys. Rev. E 85, 051914 (2012).

[31] V. Klika, R. Baker, D. Headon, and E. Gaffney, Bull. Math. Biol. 74, 935 (2012).

[32] E. A. Gaffney and S. S. Lee, Math. Med. Biol. (2013), doi:10.1093/imammb/dqt017.

[33] D. Menshykau and D. Iber, Phys. Biol. 10, 046003 (2013).

[34] S. Tanaka and D. Iber, Phys. Biol. 10, 056009 (2013). 
[35] D. Menshykau, C. Kraemer, and D. Iber, PLoS Comput. Biol. 8, e1002377 (2012).

[36] A. Badugu, C. Kraemer, P. Germann, D. Menshykau, and D. Iber, Sci. Rep. 2, 991 (2012).

[37] D. Choquet and A. Triller, Nat. Rev. Neurosci. 4, 251 (2003).

[38] J. Ries, S. R. Yu, M. Burkhardt, M. Brand, and P. Schwille, Nat. Methods 6, 643 (2009).

[39] M. Kumar, M. S. Mommer, and V. Sourjik, Biophys. J. 98, 552 (2010).

[40] B. Hebert, S. Costantino, and P. Wiseman, Biophys. J. 88, 3601 (2005).

[41] V. Parkash, V. M. Leppnen, H. Virtanen, J. M. Jurvansuu, M. M. Bespalov et al., J. Biol. Chem. 283, 35164 (2008).

[42] S. Jing, D. Wen, Y. Yu, P. L. Holst, Y. Luo et al., Cell 85, 1113 (1996).

[43] T. Spivak-Kroizman, M. Lemmon, I. Dikic, J. Ladbury, D. Pinchasi et al., Cell 79, 1015 (1994).

[44] A. N. Plotnikov, J. Schlessinger, S. R. Hubbard, and M. Mohammadi, Cell 98, 641 (1999).

[45] A. D. DiGabriele, I. Lax, D. I. Chen, C. M. Svahn, M. Jaya et al., Nature 393, 812 (1994).

[46] O. Ibrahimi, B. Yeh, A. Eliseenkova, F. Zhang, S. Olsen et al., Mol. Cell Biol. 25, 671 (2005).

[47] J. A. Goetz, S. Singh, L. M. Suber, F. J. Kull, and D. J. Robbins, J. Biol. Chem. 281, 4087 (2006).

[48] C. Scheufler, W. Sebald, M. Hlsmeyer, J. Mol. Biol. 287, 103 (1999).

[49] J. Nickel, M. K. Dreyer, T. Kirsch, and W. Sebald, J. Bone Joint Surg. 83, S7 (2001).

[50] J. B. Butt, Reaction Kinetics and Reactor Design, 2nd ed. (CRC Press, Boca Raton, FL, 2000).

[51] C. V. Pepicelli, A. Kispert, D. H. Rowitch, and A. P. McMahon, Development. Biol. 192, 193 (1997).

[52] B. C. Lu, C. Cebrian, X. Chi, S. Kuure, R. Kuo et al., Nat. Genet. 41, 1295 (2009).

[53] A. Estival, V. Monzat, K. Miquel, F. Gaubert, E. Hollande et al., J. Biol. Chem. 271, 5663 (1996).

[54] S. Ota, N. Tonou-Fujimori, N. Tonou-Fujimori, Y. Nakayama, Y. Ito et al., Genesis 48, 707 (2010).

[55] Y. Chen and G. Struhl, Cell 87, 553 (1996).

[56] M. Weaver, L. Batts, and B. L. Hogan, Development. Biol. 258, 169 (2003).

[57] R. Merino, Y. Gaan, D. Macias, A. N. Economides, K. T. Sampath et al., Development. Biol. 200, 35 (1998).

[58] J. Schnakenberg, J. Theor. Biol. 81, 389 (1979).

[59] D. Iber and D. Menshykau, Open Biol. 3, 130088 (2013).
[60] G. Celliere, D. Menshykau, and D. Iber, Biol. Open 1, 775 (2012).

[61] D. Menshykau and D. Iber, Proceedings of the COMSOL Conference (2012), arXiv:1210.0810. Paper 15375 http://comsol. co/conference2013/europe/file/id/15375/file/19959_iber_paper. pdf.

[62] J. Vollmer, D. Menshykau, and D. Iber, Proceedings of the COMSOL Conference (2013), arXiv:1309.6479. Paper 15382 https://www.comsol.com/conference2013/europe/file/id/15382/ file/19529_iber_paper.pdf.

[63] D. Bray, M. D. Levine, and C. J. Morton-Firth, Nature 397, 85 (1998).

[64] L. L. Abler, S. L. Mansour, and X. Sun, Dev. Dyn. 238, 1999 (2009).

[65] C. Chiang, Y. Litingtung, E. Lee, K. E. Young, J. L. Corden et al., Nature 383, 407 (1996).

[66] C. V. Pepicelli, P. M. Lewis, and A. P. McMahon, Curr. Biol. 8, 1083 (1998).

[67] S. Bellusci, Y. Furuta, Rush MG, R. Henderson, G. Winnier et al., Development 124, 53 (1997).

[68] K. Peters, S. Werner, X. Liao, S. Wert, J. Whitsett et al., EMBO J. 13, 3296 (1994).

[69] S. Bellusci, J. Grindley, H. Emoto, N. Itoh, and B. L. Hogan, Development 124, 4867 (1997).

[70] P. W. Ingham and A. P. McMahon, Genes Dev. 15, 3059 (2001).

[71] X. Zeng, J. A. Goetz, L. M. Suber, W. J. Scott, C. M. Schreiner et al., Nature 411, 716 (2001).

[72] A. Becskei and L. Serrano, Nature 405, 590 (2000).

[73] W. Ma, A. Trusina, H. El-Samad, W. A. Lim, and C. Tang, Cell 138, 760 (2009).

[74] P. Germann, D. Menshykau, S. Tanaka, and D. Iber, Proceedings of the COMSOL Conference (2011), arXiv:1202.0428. Paper 100817 http://www.comsol.com/paper/download/100817/ iber_paper.pdf.

[75] S. Kotha and L. Murtomäki, Math. Biosci. 253, 11 (2014).

[76] I. J. Cutress, E. J. F. Dickinson, and R. G. Compton, J. Electroanal. Chem. 638, 76 (2010).

[77] N. Sun, N. B. Wood, A. D. Hughes, S. A. M. Thom, and X. Y. Xu, Ann. Biomed. Eng. 34, 1119 (2006).

[78] Z. K. Seymen, H. Yücel, B. Karasözen, J. Comput. Appl. Math. 261, 146 (2014).

[79] B. Drawert, S. Engblom, and A. Hellander, BMC Syst. Biol. 6, 76 (2012).

[80] S. Adivarahan, D. Menshykau, O. Michos, and D. Iber, in Dynamic Image-Based Modelling of Kidney Branching Morphogenesis, Vol. 8130 of Lecture Notes in Computer Science (Springer, Berlin, 2013), pp. 106-119. 